\title{
Methylene blue decreases brain mitochondrial $A B A D$ and amyloid beta levels protecting mitochondrial functions in LPS-mouse model
}

\author{
Reham M Abdel-Kader ${ }^{*}$, Aya Zakaria, Nabila Hamdi \\ From Molecular Neurodegeneration: Basic biology and disease pathways \\ Cannes, France. 10-12 September 2013
}

\section{Background}

Methylene blue (MB) is lately being proposed to be effective in treating Alzheimer's disease (AD). Phase 2 clinical trials reported improvements in cognitive functions of $\mathrm{AD}$ patients after $\mathrm{MB}$ treatment. One of the main mechanisms of action that has been described for MB is inhibition of Tau aggregation [1]. Moreover, its antioxidant and mitochondrial protection have been previously described [2]. Only recently, a study using a triple transgenic $\mathrm{AD}$ mouse model has tested the mechanism of $M B$ in vivo, showing improved cognition and reduced $A \beta$ levels after $M B$ treatment [3].

Recently, the mitochondrial enzyme Amyloid binding alcohol dehydrogenase (ABAD) has been shown to bind $A \beta$ inducing mitochondrial dysfunction, providing a direct relation between $A \beta$ and mitochondrial dysfunction occurring in AD. Previous studies have shown that inhibiting $\mathrm{ABAD}$ protects mitochondrial functions and prevented $A \beta$-induced toxicity [4][5]. Taking into consideration the mitochondrial protective effect of $\mathrm{MB}$ and the recent data suggesting its ability to reduce $A \beta$ levels, our aim was to investigate the effect of $\mathrm{MB}$ on $\mathrm{ABAD}$ and mitochondrial function in an LPS mouse model that has been previously described to induce memory impairment, with $A \beta$ accumulation in hippocampus and cerebral cortex [6].

\section{Materials and methods}

LPS mouse model was used (I.P $250 \mu \mathrm{g} / \mathrm{kg}$ LPS for 7 consecutive days) and accumulation of $A \beta$ was assessed by immunohistochemistry. To Test the effect of MB on this in vivo model, mice were treated for 4 days with MB (I.P 4 $\mathrm{mg} / \mathrm{kg}$ ). After sacrification of the mice, mitochondrial associated ROS and cell viability of brain cells were measured using dihydrorhodamine probe and MTT assay

$$
\text { Department of Pharmacology \& Toxicology, German University in Cairo, }
$$$$
\text { Cairo, Egypt }
$$

respectively. Moreover, $A B A D$ and $A \beta$ levels were determined by western blotting in the brains of the treated mice compared to the control group.

\section{Results}

The LPS mouse model used in this study showed brain accumulation of $A \beta$, decreased cell viability and increased mitochondrial associated ROS levels compared to the vehicle group. MB treatment significantly increased cell viability and reduced the LPS-induced increase in the ROS level. Moreover, brain $A \beta$ level of the MB treated group was significantly decreased compared to the untreated group. Most interestingly, MB treatment was able to reduce the high level of ABAD that was found in the LPS mouse model.

\section{Conclusions}

Taken together our results showed that MB decreased both $A \beta$ and $A B A D$ levels, while protecting the mitochondria from oxidative stress, consequently improving brain cell viability. Based on the previously reported role of the interaction between $A B A D$ and $A B$ in inducing mitochondrial dysfunction, the current study suggests a novel mechanism of action of $\mathrm{MB}$, linking its mitochondrial protective effects to its lowering effect of both $\mathrm{AB}$ and $\mathrm{ABAD}$ which may decrease their binding and the resultant mitochondrial stress.

Published: 13 September 2013

doi:10.1186/1750-1326-8-S1-P1

Cite this article as: Abdel-Kader et al:: Methylene blue decreases brain mitochondrial ABAD and amyloid beta levels protecting mitochondrial functions in LPS-mouse model. Molecular Neurodegeneration 2013 8(Suppl 1):P1 